\title{
Scale-dependent roughening kinetics in vapor deposited gold
}

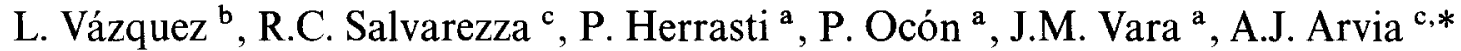 \\ ${ }^{a}$ Departamento de Quimica Física Aplicada C-II, Universidad Autónoma de Madrid, 28049 Madrid, Spain \\ ${ }^{\mathrm{b}}$ Instituto de Ciencia de Materiales, CSIC, Departamento Fisica Aplicada, C-XII, Universidad Autónoma de Madrid, 28049 Madrid \\ Spain \\ ' Instituto de Investigaciones Fisicoquímicas Teóricas y Aplicadas (INIFTA), Sucursal 4, Casilla de Correo 16, (1900) La Plata, \\ Argentina
}

Received 14 June 1995; accepted for publication 8 August 1995

\begin{abstract}
The roughening kinetics of gold deposits grown from vapor was studied by scanning tunneling microscopy. The dynamic scaling yielded the following growth exponents $\alpha(\mathrm{I})=0.90 \pm 0.06$ and $\beta(\mathrm{I})=0.25 \pm 0.06$ for $L_{\mathrm{s}}<d_{\mathrm{s}}$, and $\alpha(\mathrm{II})=0.37 \pm 0.05$ and $\beta(\mathrm{II})=0.45 \pm 0.06$ for $L_{\mathrm{s}}>d_{\mathrm{s}}$, where $L_{\mathrm{s}}$ is the scan length and $d_{\mathrm{s}}$ is the average diameter of columns. The scaling properties of the domain-dependentsurface roughness exponents allowed us to give the rationale for experimental data on the fractal behavior of thin metal films.
\end{abstract}

Keywords: Computer simulations; Gold; Metallic films; Scanning tunneling microscopy; Surface roughening

\section{Introduction}

Aggregation processes involved in the growth of a solid phase on a foreign flat solid substrate play a key role in the dynamics and stability of interfaces. In general, the macroscopic shape of a growing object always implies a roughness of its surface. To characterize the roughness of growing surfaces the concept of statistical scale invariance has been introduced [1]. This concept is involved in the more general approach of fractal geometry [2].

Both the concept of statistical scale invariance and fractal geometry are applicable to real systems only within certain scale limits which are fine enough to capture the stochastic nature of the growth process, but sufficiently coarse to allow us

\footnotetext{
* Corresponding author. Fax: +54 21254642 .
}

to ignore the discrete lattice structure, overhang formation and other microscopic details.

In general, the average shape of the growing object is not explicitly known, and therefore the study of shape fluctuations is not possible [1]. This drawback can be circumvented by considering the geometry of the substrate as an infinite $d$ dimensional plane in a $(d+1)$-dimensional space. Then, at time $t$, the surface configuration can be described as a single-valued, continuous function, $h(x, t)$ which is a measure of the object height perpendicular to the substrate at the point $x$. The function $h(x, t)$ represents a random function which fulfills the condition $\langle h(x, t)\rangle=0$. The growth process is statistically invariant provided that by suitable rescaling $x, t$, and $h$, typical surface configurations can be made "to look the same" [1].

The phase growth kinetics can be followed by means of the dynamic scaling theory applied to 
surface profiles on different time scales [1]. Thus, for a surface profile of length $L$ consisting of $N$ points, the theory predicts that $\xi(L,\langle h\rangle)$, the interface width, and $\langle h\rangle$, the average profile thickness or height, scale as [1]

$\xi(L,\langle h\rangle) \propto L^{\alpha} f(x)$,

where $\xi(L,\langle h\rangle)$ is defined by

$\xi(L,\langle h\rangle)=\left[1 / N \sum\left[h\left(x_{i}\right)-\langle h\rangle\right]^{2}\right]^{1 / 2}$,

and $h\left(x_{i}\right)$ is the deposit height measured along the $x$-direction at the point $x_{i}$, and $x=\langle h\rangle / L^{z}$, with $z=\alpha / \beta, \beta$ and $\alpha$ being the dynamic and static growth exponents, respectively. Furthermore, $f(x)$ has the following properties: $f(x)=$ const for $x \Rightarrow \infty$, and $f(x)=x^{\beta}$ for $x \Rightarrow 0$. Eq. (1) comprises two limiting situations, namely,

$\xi(\langle h\rangle) \propto\langle h\rangle^{\beta}$,

for $\langle h\rangle \Rightarrow 0$, and

$\xi(L) \propto L^{\alpha}$,

for $\langle h\rangle \Rightarrow \infty$. Exponents $\alpha$ and $\beta$, and the ratio $\alpha / \beta$ contain information about the scaling properties of the surface fluctuations, $\alpha$ covering the range $0<\alpha<1$. Otherwise, $\beta$ describes the increase of surface roughness with time through Eq. (3). Static and dynamic surface exponents are coupled by the growth process itself through the scaling relation $z=\alpha / \beta[1]$. The exponent $z$ reflects the temporal spread of the surface fluctuations, and its reciprocal has been termed the coarsening exponent.

Atomistic-type growth models such as the Eden [3], ballistic deposition [4] and restricted solidon-solid models [5] have been proposed to explain non-equilibrium growth processes. These models can be successfully described by the Kardar, Parisi and Zhang motion equation [6] which leads to $\alpha=0.40$ and $\beta=0.25$ in 3d-growth, and $\alpha+\alpha / \beta=2$ in all dimensions. However, for a number of growth processes the KPZ model does not appear to be the most adequate, in contrast to those models based on nonlinear diffusion equations which in $3 \mathrm{~d}$ yield either $\alpha=1.0$ and $\beta=0.25[7,8]$ or $\alpha=$ 0.66 and $\beta=0.24$ [9].

Attempts have recently been made to determine the surface properties of different thin films using scanning probe microscopies, adsorption, and
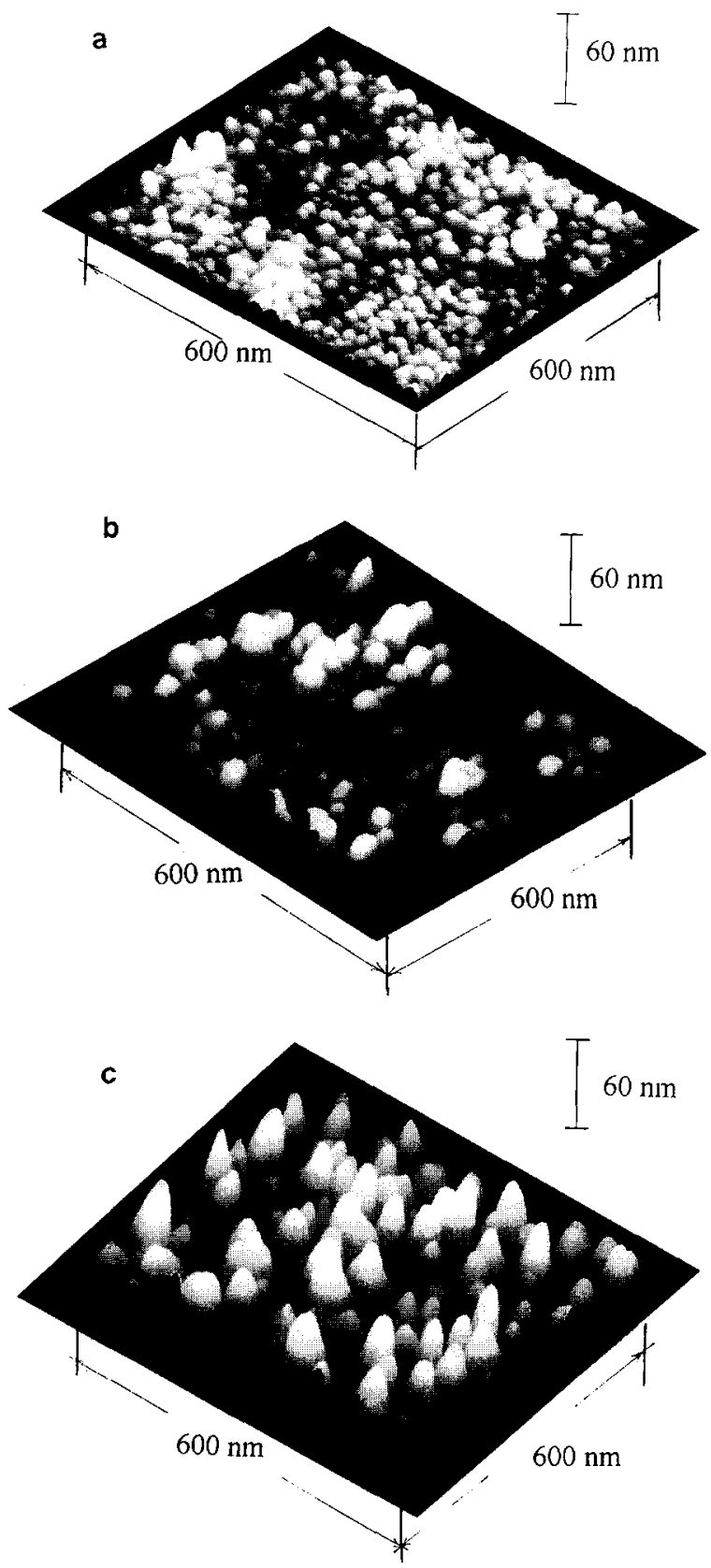

Fig. 1. (a)-(c). $600 \times 600 \mathrm{~nm}^{2} 3 \mathrm{~d}$ STM images of a vapor deposited gold film grown at $v=0.2 \mathrm{~nm} \mathrm{~s}^{-1}$ and $T_{\mathrm{s}}=298 \mathrm{~K}$. (a) $\langle h\rangle=27 \mathrm{~nm}$; (b) $\langle h\rangle=270 \mathrm{~nm}$; (c) $\langle h\rangle=1140 \mathrm{~nm}$. STM images have the same $z$-scale. The bar indicates $60 \mathrm{~nm}$. (d) The 3d STM image (upper) and the corresponding cross section (lower) show the characteristics of column tips of a vapor deposited gold film, $\langle h\rangle=1140 \mathrm{~nm}$. (e) $\log _{10} d_{3}$ versus $\log _{10}\langle h\rangle$ plot 

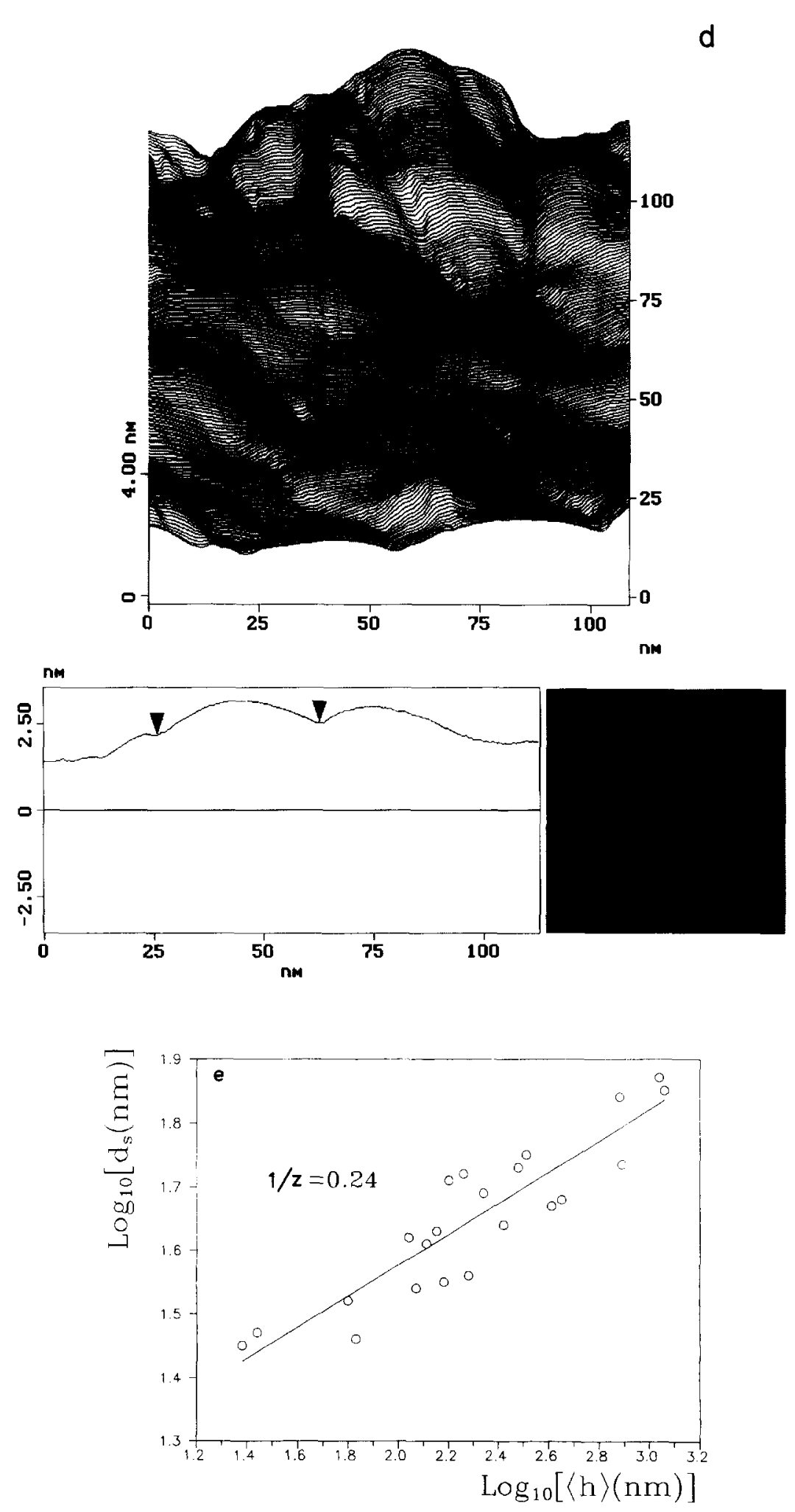

Fig. 1 (continued). 
$X$-ray reflectivity [10-19]. In all cases, it has been found that these surfaces can be described as selfaffine fractals, although quite different values of $\alpha$ and $\beta$ have been reported (Table 1). Some of these values of $\alpha$ and $\beta$ seem to agree with the predictions of the KPZ model, but when both exponents could be obtained, the $\alpha+(\alpha / \beta)=2$ relationship was not obeyed $[13,14]$. In other cases, the values of $\alpha$ and $\beta$ were close to those resulting from growth models incorporating surface diffusion. However, except for data reported in Refs. [10-12], it still remains unclear whether $\alpha$ actually refers to the steady surface roughness regime as required by the theory [1]. In addition, for different systems it is difficult to compare the $\alpha$ and $\beta$ values obtained at different length scales owing to the appearance of cutoffs which confine the self-affine fractal behavior of thin solid films [10-12]. Thus, despite the increasing number of investigations on the matter a coherent view of data is still lacking.

In this paper we report scanning tunneling microscopy (STM) imaging of vapor deposited gold films grown under non-equilibrium conditions over a broad range of thickness. The dynamic scaling analysis applied to STM profiles leads to three characteristic sets of surface roughness exponents, depending on the range of $L_{\mathrm{s}}$. Thus, for $L_{\mathrm{s}}<d_{\mathrm{s}}$, the average diameter of the columns, $\alpha(\mathrm{I})=$ 0.90 and $\beta(\mathrm{I})=0.25$, for $d_{\mathrm{s}} \leqslant L_{\mathrm{s}} \leqslant 500 \mathrm{~nm} \alpha(\mathrm{II})=$ 0.37 and $\beta(\mathrm{II})=0.45$, and for $L_{\mathrm{s}}>500 \mathrm{~nm} \alpha \cong 0$, i.e.

Table 1

Values of $\alpha$ and $\beta$ from the literature

\begin{tabular}{|c|c|c|c|c|}
\hline Film & $\alpha$ & $\beta$ & Region & Reference \\
\hline Vapour deposited & 0.36 & - & $L>d_{\mathrm{s}}$ & {$[8-10]$} \\
\hline Gold & 0.9 & - & $L<d_{s}$ & {$[8-10]$} \\
\hline $\begin{array}{l}\text { Sputtered deposited } \\
\text { Gold }\end{array}$ & 0.4 & 0.4 & - & {$[11]$} \\
\hline Evaporated & 0.7 & 0.26 & $L<d_{\mathrm{s}}$ & {$[12,13]$} \\
\hline Silver & 0.5 & - & - & {$[14]$} \\
\hline Vapour deposited & 0.79 & 0.22 & - & {$[15]$} \\
\hline Iron & - & $0.22-0.32$ & - & {$[16]$} \\
\hline Cuprous chloride & 0.84 & - & - & {$[17]$} \\
\hline Copper & 1.0 & $0.5-0.25$ & & {$[26]$} \\
\hline
\end{tabular}

$d_{\mathrm{s}}$ is either the average grain size or the average column diameter. the interface width saturates. These results make it possible to give the rationale for the self-affine fractal behavior of thin metal film surfaces recently reported $[10,14,20]$.

\section{Experimental}

Gold films were grown from the vapor phase on a smooth glass substrate employing an evaporator chamber at pressure $P=10^{-4}$ Torr, average deposit growth rate $v=0.2 \mathrm{~nm} \mathrm{~s}^{-1}$, and substrate temperature $T=298 \mathrm{~K}$ [10-12]. The angle between the direction of incident particles and the substrate normal was set in the range $2-25^{\circ}$. Values of $\langle h\rangle$ in the range $27 \mathrm{~nm}<\langle h\rangle<1140 \mathrm{~nm}$ were measured with a profilometer. Gold films prepared in this way are described as columnar structured, polycrystalline films in which columns exhibit a (111) preferred crystallographic orientation [12]. Details about the experimental procedure have been reported elsewhere [10-12].

The topography of gold deposits was imaged with a Nanoscope III STM operating in air. STM tips were made from $0.25 \mathrm{~mm}$ dia platinum wires directly by cutting. STM measurements were made using a $0.05 \mathrm{~V}$ bias voltage with the tip $(+)$ at 1-2 nA constant current. Different tips were used to discard occasional artifacts caused by the tip geometry. To minimize errors [9] STM images with $512 \times 512$ pixels were taken. STM data were analyzed after fitting the instrument plane and applying a subtracting procedure [20]. In all cases $\xi_{\mathrm{stm}}^{\mathrm{m}}$, the interface width of the deposit resulting from STM scans, was also corrected for $\xi_{\mathrm{g}}$, the interface width of the glass substrate. The value of $\xi_{\mathrm{g}}$ was determined by atomic force microscopy $\left(\right.$ AFM). Thus, values of $\xi_{\text {stm }}=\left(\xi_{\mathrm{stm}}^{\mathrm{m} 2}-\xi_{\mathrm{g}}^{2}\right)^{1 / 2}[14]$ were used to evaluate $\alpha$ and $\beta$. However, as AFM and STM would provide different height informations, AFM measurements on the gold films were also made yielding similar height values from both techniques.

\section{Results and discussion}

Vapor deposited gold films for increasing values of $\langle h\rangle$ (Figs. 1a-1c) developed a progressive rough- 
ening resulting from the competitive columnar growth. The value of $d_{\mathrm{s}}$, which corresponds to the average diameter of the columnar tips, measured from the STM images (Fig. 1d) increased with $\langle h\rangle$ as $d_{\mathrm{s}} \propto\langle h\rangle^{p}$ with $p=0.24 \pm 0.06$ (Fig. 1e). Likewise, for $\langle h\rangle\left\langle 500 \mathrm{~nm}, \xi_{\text {stm }}\right.$ increased with $\langle h\rangle$ (Fig. 2), whereas for $\langle h\rangle>500 \mathrm{~nm}$, a steady state roughness was reached. It should be noted that the columnar surface itself is rather smooth as shown in the cross section depicted in Fig. 1d.

Accurate values of $\alpha$ were obtained by using the single image dynamic scaling method $[10,11,21]$. Accordingly, $\alpha$ resulted from the steady roughness regime equation

$$
\xi_{\text {stm }}\left(L_{s},\langle h\rangle\right) \propto L_{s}^{\alpha},
$$

where $L_{\mathrm{s}}$ corresponds to the segment of the STM scan of size $S$ measured in the $x$-direction. Therefore, the magnitude $L_{\mathrm{s}}$ resulting from the STM scan corresponds to $L$ in Eq. (1). For each scan 486 pairs of data points $\left(L_{\mathrm{s}}, \xi_{\mathrm{stm}}\right)$ have been obtained, $L_{\mathrm{s}}$ being varied from $S / 128$ to $0.96 \times S$. Finally, for each $L_{\mathrm{s}}$ the value of $\xi_{\text {stm }}$ is the average of 512 scans of the same image, and the $\log _{10} \xi_{\text {stm }}$ versus $\log _{10} L_{\mathrm{s}}$ plot is obtained (Fig. 3 ). This plot for a gold deposit with $\langle h\rangle=1105 \mathrm{~nm}$, exhibits two linear regions with a crossing point at $\log _{10} L_{\mathrm{sc}} \cong 1.6$, and a saturation region for $\log _{10} L_{\mathrm{s}}>2.6$. The slopes of the straight lines are $\alpha(\mathrm{I})=0.83 \pm 0.03$ for $\log _{10} L_{\mathrm{s}}<1.6$, and $\alpha(\mathrm{II})=$ $0.37 \pm 0.02$ for $\log _{10} L_{\mathrm{s}}>1.6$, with the crossover at $L_{\mathrm{sc}} \cong 35 \mathrm{~nm}$, a figure which is close to $d_{\mathrm{s}} / 2$. Similarly, after averaging more than 15 different STM images it resulted in $\langle\alpha(I)\rangle=0.89 \pm 0.07$ and $\langle\alpha(\mathrm{II})\rangle=0.37 \pm 0.05$. These results were also confirmed by using the multiple image dynamic scaling method $[12,20,21]$ through the relationship

$\xi_{\text {stm }} \propto S^{\alpha}$.

On the other hand, the exponent $\beta$ can be evaluated from Eq. (3) considering $\xi_{\text {stm }}$ and $\langle h\rangle$ data for $\langle h\rangle\left\langle L_{\mathrm{s}}\right.$. Accordingly, the $\log _{10} \xi_{\text {stm }}$ versus $\log _{10}\langle h\rangle$ plot resulting from 15 STM images for each $\langle h\rangle$, reveals straight lines (Fig. 4) with $L_{s}$ dependent slopes $\langle\beta\rangle$. Thus, for $\langle h\rangle\left\langle L_{\mathrm{s}}\right.$ the value of $\langle\beta\rangle$ converges to $\langle\beta(\mathrm{II})\rangle=0.45 \pm 0.06$ (Fig. 5). In principle, the value $\langle\beta(\mathrm{I})\rangle$ cannot be derived from these plots for $\langle h\rangle \geqslant d_{\mathrm{s}} / 2 \cong L_{\mathrm{sc}}$ as $\langle h\rangle$ should be much smaller than $L_{\mathrm{s}}[1]$. Then, $\langle\beta(\mathrm{I})\rangle$ was estimated from the relationship $d_{\mathrm{s}} \propto\langle h\rangle^{p}$ as

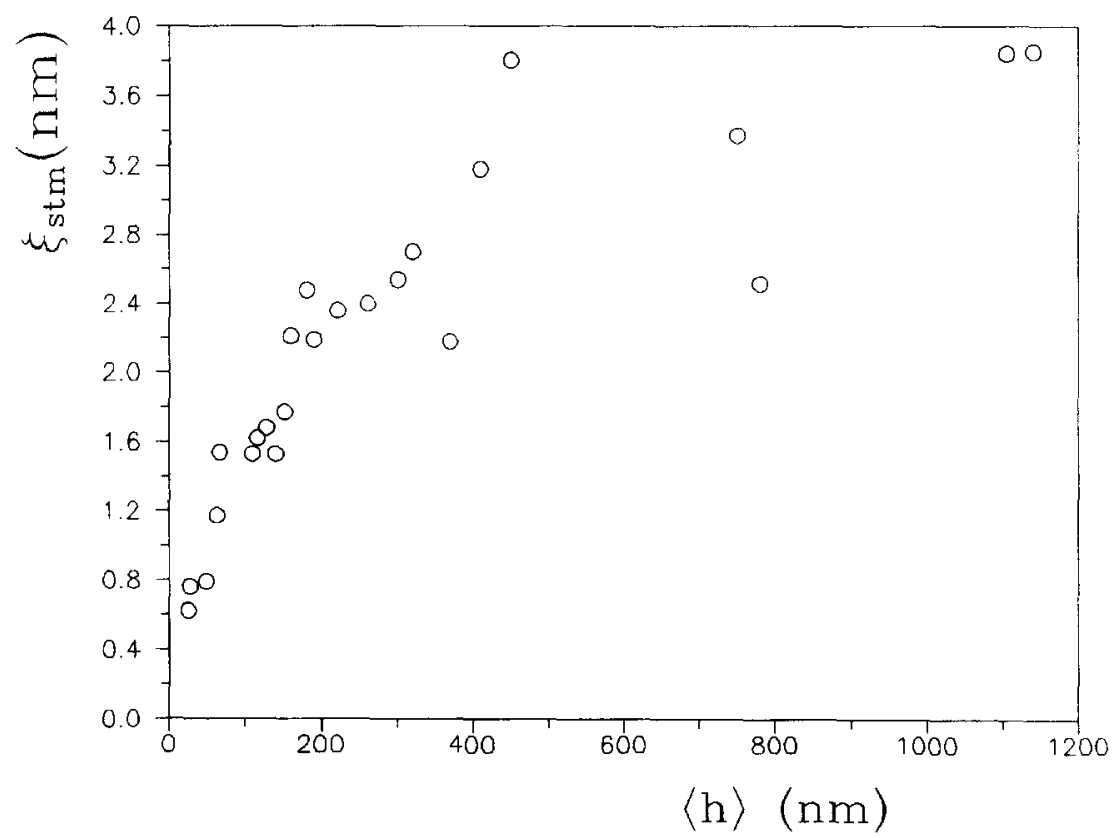

Fig. 2. $\xi_{\text {stm }}$ versus $\langle h\rangle$ plot. 


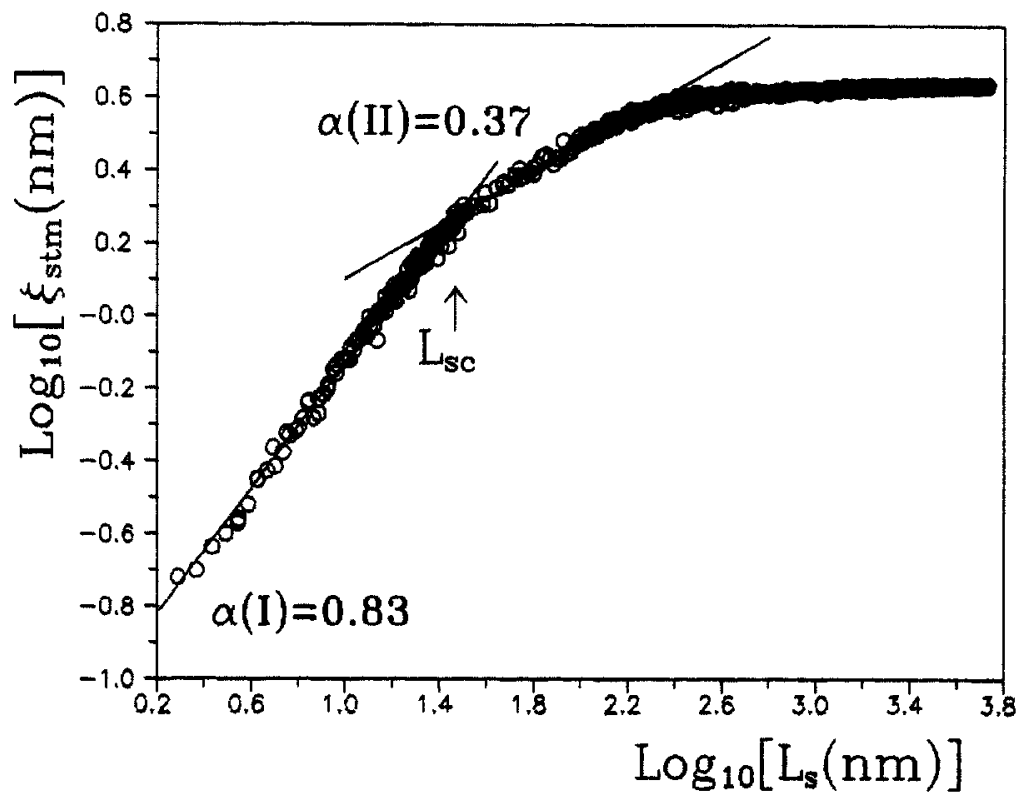

Fig. 3. $\log _{10} \xi_{\text {stm }}$ versus $\log _{10} L_{\mathrm{s}}$ plot for vapor deposited gold films grown at $v=0.2 \mathrm{~nm} \mathrm{~s}^{-1}$ and $T_{\mathrm{s}}=298 \mathrm{~K}$. The interface is in the steady state roughness regime, $\langle h\rangle=1105 \mathrm{~nm}$. The value of $L_{\mathrm{sc}}$ is close to $d_{\mathrm{s}} / 2$.

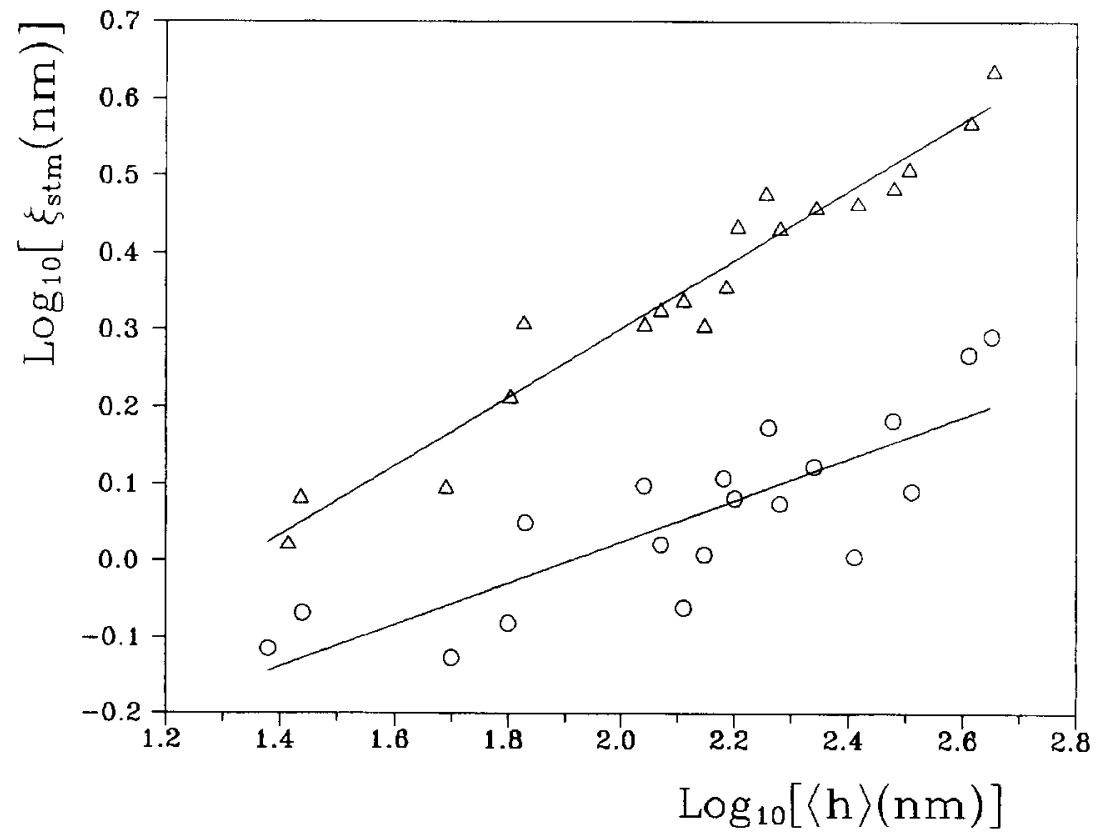

Fig. 4. $\log _{10} \xi_{\mathrm{atm}}$ versus $\log _{10}\langle h\rangle$ plots $\left(\langle h\rangle\langle 400 \mathrm{~nm})\right.$ at different $L_{\mathrm{s}}$ values for vapor deposited gold films grown at $v=0.2 \mathrm{~nm} \mathrm{~s}$ and $T_{\mathrm{s}}=298 \mathrm{~K}$. (O) $L_{\mathrm{s}}=26 \mathrm{~nm}$, the slope is $0.27 ;(\triangle) L_{\mathrm{s}}=600 \mathrm{~nm}$ with a slope of 0.45 . 


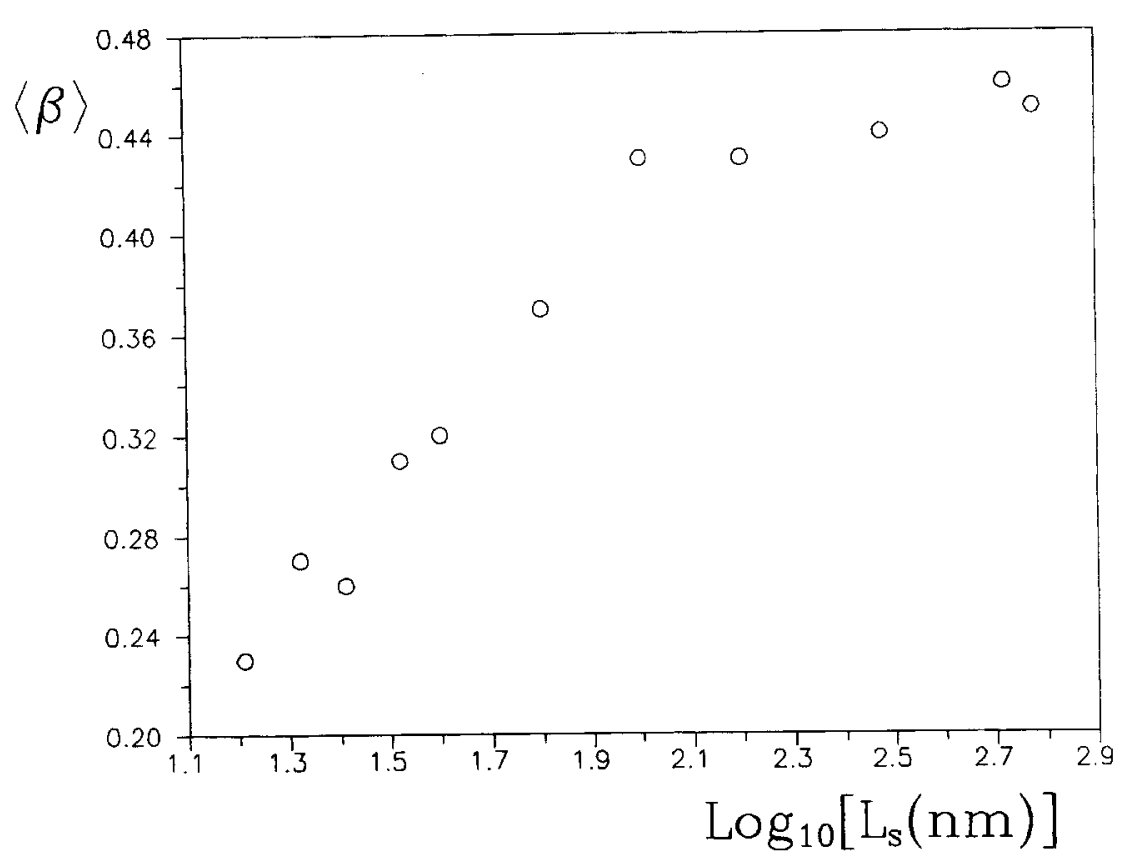

Fig. 5. $\langle\beta\rangle$ versus $\log _{10} L_{\mathrm{s}}$ plot. The domain size dependence of $\beta$ is concluded from this plot.

$p=\beta / \alpha[1,14]$. Hence, taking $p \cong 0.24$ (Fig. 1d) and $\langle\alpha(\mathrm{I})\rangle=0.89$, it results in $\langle\beta(\mathrm{I})\rangle=0.22 \pm 0.06$. These figures can explain why $\beta \Rightarrow\langle\beta(\mathrm{I})\rangle$ for $\log _{10} L_{\mathrm{s}} \Rightarrow 1.2$ as the condition $h \ll L^{\alpha / \beta}$ is fulfilled in the overall range of $\langle h\rangle$ (Fig. 5).

It should be noted that computer simulated fractals provide data covering four to five orders of magnitude as usually required for logarithmic fitting, in contrast to experimental systems for which this situation is less ambitious owing to the existence of inner and outer cutoffs. Therefore, $\log _{10} \xi_{\text {stm }}$ versus $\log _{10} L_{\mathrm{s}}$ linear plots covering at least one order of magnitude or thereabouts have to be considered as acceptable $[14,20]$.

The values of $\alpha$ and $\beta$ for $L_{\mathrm{s}}<d_{\mathrm{s}}$ can be related to $\alpha=0.9-0.7[10-11,14-17,19]$ and $\beta=0.22-0.32$ $[14,17-18]$ reported in the literature for other systems where seemingly the scaling analysis was restricted to values of $L_{\mathrm{s}}$ smaller than the size of the elements which form the film.

On the other hand, for $d_{\mathrm{s}}<L_{\mathrm{s}}<500 \mathrm{~nm}$ $\alpha(\mathrm{II}) \cong 0.37$ and $\beta=0.45$. These figures appear to be consistent with $\alpha=0.4$ [10-13] and $\beta=0.4$ [13] previously reported for other thin film systems.

The physical origin of the two regions in the $\log _{10} \xi_{\text {stm }}$ versus $\log _{10} L_{\mathrm{s}}$ plots can be assigned to different domains in the columnar structure which are revealed by changing the scale length, i.e. the microscope resolution. The first domain which is restricted to a scale length in the order of the column size, corresponds to scaling properties at single column surfaces. The second domain which appears at scale lengths larger than the average columnar size can be related to column height fluctuations. This interpretation can be supported by scaling computer simulated surfaces resulting from an ensemble of columns fluctuating in height with known values of $\alpha_{\mathrm{s}}$ (Figs. 6a-6c). The surface of each column is represented by a smooth rounded cusp of diameter $d_{s}$. For this surface model the $\log _{10} \xi$ versus $\log _{10} L$ plots exhibit one or two linear regions depending on the $L / d_{\mathrm{s}}$ ratio. For $L / d_{\mathrm{s}} \gg 1$ (low resolution limit, Fig. 6a) only one region with slope $\alpha_{\mathrm{s}}$ is observed (Fig. 6d plot A). Otherwise, for $L / d_{\mathrm{s}} \cong 20$ (Fig. 6b) two linear regions with $\alpha \Rightarrow 1$ for $L<d_{\mathrm{s}}$, and $\alpha=\alpha_{\mathrm{s}}$ for $L_{\mathrm{s}}>d_{\mathrm{s}}$ are observed (Fig. 6d plots B). Finally, for $L / d_{\mathrm{s}} \Rightarrow 1$ (high resolution limit) (Fig. 6c) only the region with $\alpha \cong 1$ results from the $\log _{10} \xi$ versus $\log _{10} L$ plots. Note that $\alpha \cong 1$ is consistent with a smooth 
rounded cusp surface. In addition, for a given $L$, the value of $L_{\mathrm{sc}}$ increases as $d_{\mathrm{s}}$ is increased. This is an indication that the crossover can be taken as a measure of the average diameter of the columns.

The preceding explanation is also consistent with recent data on the self-affine characteristics of vapor deposited gold grown in the range 298-673 K where surface diffusion dominates [22]. Thus, as the temperature is increased, and accordingly the average column size increases, the film topography with $\alpha(\mathrm{I})=0.9$ prevails and the topography with $\alpha(\mathrm{II}) \cong 0.4$ tends to disappear.

Experimental data on $L_{\mathrm{s}}<d_{\mathrm{s}}$ show that the surface of the deposit approaches smoothness, therefore it is reasonable to admit that surface diffusion would play a key role in determining the topography of the deposit [23]. However, as recently reported [24], simple surface diffusion eventually favours the formation of grooves leading to large values of $\beta$, in contrast to $\beta=0.25$, as reported in this work. On the other hand, growth models for unstable growth with a Schwöbel barrier predict a pyramid-like surface profile with either $\alpha=1$ and $\beta=0.5$ [25] or $\alpha=1$ and $\beta<0.5$ [26], depending on whether the interlayer mass transport is either completely or partially inhibited. Certainly, one may speculate that, as it occurs for $\mathrm{Cu}$ at low temperature [25], the barrier for gold at $298 \mathrm{~K}$ is still sufficiently low to allow interlayer mass transport, as it could be concluded from $\beta=$ 0.25 . It should also be noted that the values $\alpha=1$ and $\beta=0.25$ derived from smooth pyramids should be considered as effective exponents rather than true scaling exponents [8] because of the euclidean characteristics of the surface deposit. In principle, this could also be the case in our work as rounded and smooth column tips approach a euclidean surface. However, there are some important differences between data and the expectations of the model given in Ref. [8]. In fact, in this work we are dealing with a columnar rather than a pyramidlike structure, and the slope of the elements in the columnar structure is much weaker (Fig. 1d) than that expected for the model referred to in Ref. [8]. Furthermore, the experimental value of $\alpha$ is 0.9 rather than 1 . Accordingly, the scaling description of the gold deposit for $L_{\mathrm{s}}<d_{\mathrm{s}}$ appears to be valid.

On the other hand, the roughness exponents

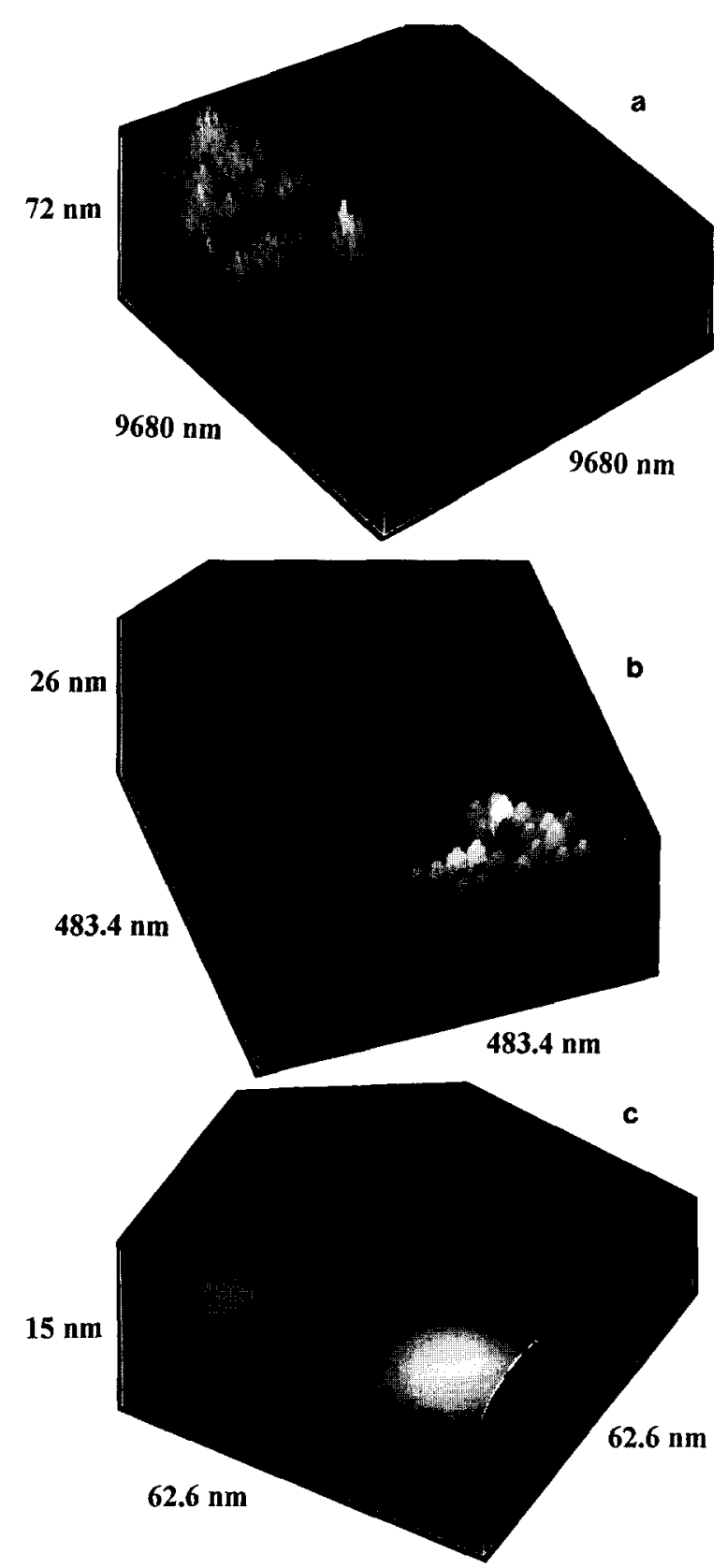

Fig. 6. (a)-(c) 3D-images of computer simulated surfaces consisting of fluctuating height columns with $\alpha_{\mathrm{s}}=0.4$ and $d_{\mathrm{s}}=$ $24.2 \mathrm{~nm}$ as described in the text. (a) $9680 \times 9680 \mathrm{~nm}^{2}, L / d_{\mathrm{s}}=$ 400 ; (b) $483.4 \times 483.4 \mathrm{~nm}^{2}, L / d_{s}=20$; (c) $62.6 \times 62.6 \mathrm{~nm}^{2}, L / d_{\mathrm{s}}=$ 3. (d) $\log _{10} \xi$ versus $\log _{10} L$ plots resulting from the scaling of simulated surfaces. Plot A $\left(^{*}\right), L=9680 \mathrm{~nm}, d_{s}=24.2 \mathrm{~nm}$ and $L / d_{\mathrm{s}}=400$. Plot $\mathrm{B}(\triangle), L=483.4 \mathrm{~nm}, d_{\mathrm{s}}=24.2 \mathrm{~nm}$, and $L / d_{\mathrm{s}}=20$. 


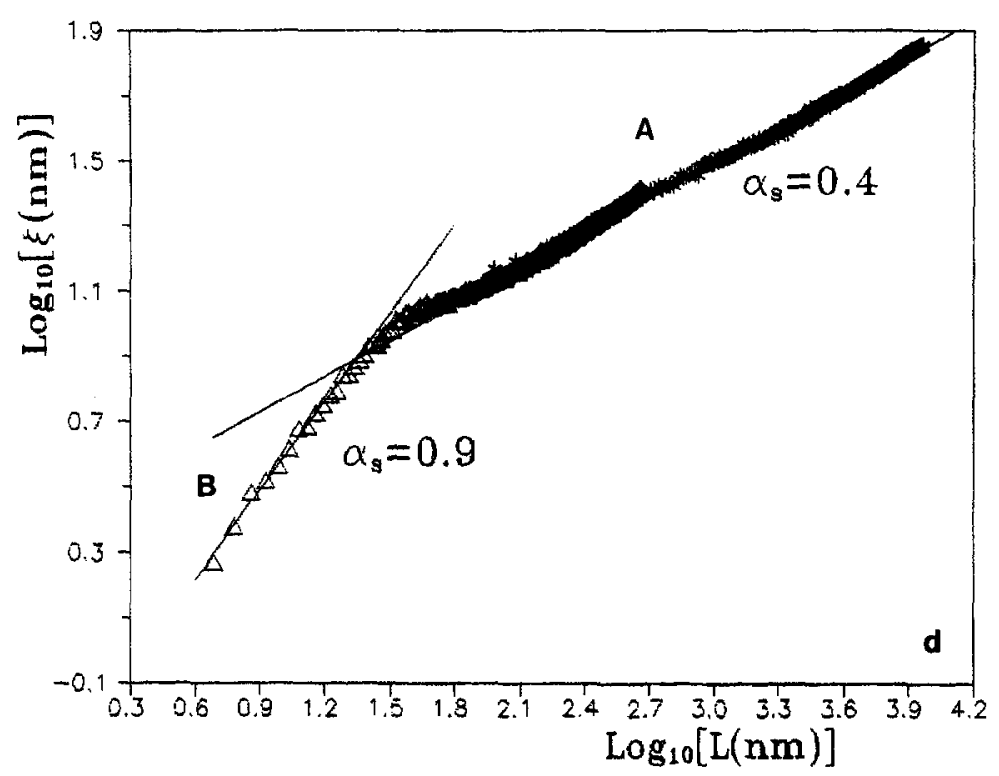

Fig. 6 (continued).

reported in this work for $L_{\mathrm{s}}<d_{\mathrm{s}}$, as well as those published in Ref. [26], coincide with the roughness exponents derived from growth models based on the linear equation diffusion [27]. This could be taken as an indication that the kinetics of the growth process, under the conditions of this work, is governed by this equation.

Finally, for $L_{\mathrm{s}}>d_{\mathrm{s}}$ one obtains $\alpha=0.4$ and $\beta=$ 0.4 , which describe the evolution of fluctuations between growing columns. While the value of $\alpha$ is close to that predicted by the KPZ model, the experimental $\beta$ value is substantially larger than the theoretical value $\beta=0.25$, as it has been already reported for other experimental systems [13]. In this case an unambiguous interpretation of these exponents in terms of existing growth models is not feasible.

Changes in the growth exponents with the scale length have been predicted from computer simulations of growing columns [28], whereas for experimental systems only qualitative changes in the surface topography according to the scale length have been reported [29]. Our results have demonstrated for the first time, that both growth exponents for thin gold evaporated films depend on the scale length where the scaling analysis is performed. This fact is useful to understand apparent discrepancies of those exponents reported for different systems and the physical processes involved in the roughening of metal surfaces [30].

\section{Acknowledgements}

This work was financially supported by the Consejo Nacional de Investigaciones Cíentíficas y Técnicas of Argentina (CONICET) and Consejo Superior de Investigaciones Cíentíficas of Spain (CSIC) under a scientific cooperation agreement.

\section{References}

[1] F. Family and T. Vicsek (Eds.), Dynamics of Fractal Surfaces (World Scientific, Singapore, 1991);

F. Family, Physica A 168 (1990) 561, and references therein.

[2] B. Mandelbrot, The Fractal Geometry of Nature, (Freeman, San Francisco, 1982).

[3] M. Eden, Proc. 4th Berkeley Symp. on Mathematical Statics and Probability, F. Neyman, Vol. 4 (Univ. of California Press, Berkeley, 1961).

[4] P. Meakin, P. Ramanlal, L.M. Sander and R.C. Ball, Phys. Rev. A 34 (1986) 5091

[5] H.C. Kang and J.W. Evans, Surf. Sci. 271 (1992) 321.

[6] M. Kardar, G. Parisi and Y.C. Zhang, Phys. Rev. Lett. 56 (1986) 889.

[7] J. Villain, J. Phys. I 1 (1992) 19. 
[8] M. Siegert and M. Plischke, Phys. Rev. Lett. 73 (1994) 1517.

[9] Z.W. Lai and S. Das Sarma, Phys. Rev. Lett. 66 (1991) 2348.

[10] R.C. Salvarezza, L. Vázquez, P. Herrasti, P. Ocón, J.M. Vara and A.J. Arvia, Europhys. Lett. 20 (1992) 727.

[11] L. Vázquez, R.C. Salvarezza, P. Herrasti, P. Ocón, J.M. Vara and A.J. Arvia, Appl. Surf. Sci. 70/71 (1993) 413.

[12] P. Herrasti, P. Ocón, L. Vázquez, R.C. Salvarezza, J.M. Vara and A.J. Arvia, Phys. Rev. A 45 (1992) 7440.

[13] H. You, R.P. Chiarello, H.K. Kim and K.G. Vandervoort, Phys. Rev. Lett. 70 (1993) 2900.

[14] C. Thompson, G. Palasantzas, Y.P. Feng, S.K. Sinha and J. Krim, Phys. Rev. B 49 (1994) 4902; P. Pfeifer, Y.J. Wu, M.W. Cole and J. Krim, Phys. Rev. Lett. 62 (1989) 1997.

[15] M.V. Rao, B.K. Mathur and K.L. Chopra, Appl. Phys. Lett. 65 (1994) 124

[16] R. Chiarello, V. Panella, J. Krim and C. Thompson, Phys. Rev. Lett. 67 (1991) 3408.

[17] Y.L. He, H.N. Yang and T.M. Lu, Phys. Rev. Lett. 69 (1992) 3770.

[18] J. Chevrier, V. Le Thanh, R. Buys and J. Derrien, Europhys. Lett. 16 (1991) 737.
[19] W.M. Tong, R. Stanley Williams, A. Yanase, Y. Segawa and M.S. Anderson, Phys. Rev. Lett. 72 (1994) 3374.

[20] J. Krim, I. Heyvaert, C. Van Haesendonck and Y. Bruynseraede, Phys. Rev. Lett. 70 (1993) 57.

[21] L. Vázquez, R.C. Salvarezza, P. Herrasti, P. Ocón, J.M. Vara and A.J. Arvia, Chaos, Solitons and Fractals 6 (1995) 569.

[22] L. Zubimendi, M.E. Vela, R.C. Salvarezza, L. Vázquez, J.M. Vara and A.J. Arvia, Phys. Rev. E 50 (1994) 1367.

[23] J.A. Stroncio and D.T. Pierce, Phys. Rev. B 49 (1994) 8522.

[24] F. Family, in: Fractals in the Natural and Applied Sciences, Ed. M.M. Novak (North-Holland, Amsterdam, (1994) p. 1

[25] Z. Zhang, J. Decht and H. Metiu, Phys. Rev. B 48 (1993) 4972.

[26] H.J. Ernst, F. Fabre, R. Folkerts and J. Lapujoulade, Phys. Rev. Lett. 72 (1994) 112.

[27] D.E. Wolf and J. Villain, Europhys. Lett. 13 (1992) 389.

[28] J. Krug and P. Meakin, Phys. Rev. A 43 (1991) 900.

[29] M.D. Johnson, C. Orme, A.W. Hunt, D. Graff, J. Sudijono, L.M. Sander and B.G. Orr, Phys. Rev. Lett. 72 (1994) 116.

[30] J. Lapujoulade, Surf. Sci. Rep. 20 (1994) 1. 